\title{
Two-Domain Model for Orthogonal Fluxgate
}

\author{
Mattia Butta and Pavel Ripka \\ Czech Technical University, Faculty of Electrical Engineering, Prague 166 27, Czech Republic
}

In this paper a new model for orthogonal fluxgate is presented. A first attempt to explain the working principle of the orthogonal fluxgates was done in the 1970's. We show that this model does not work well on recently developed orthogonal fluxgate sensors with thin-film core on microwire. A new more accurate two-domain model based both on domain wall motion and magnetization rotation is proposed. We show that the new model better explains the observed properties of thin-film orthogonal fluxgate.

Index Terms-Magnetic field measurement, model, orthogonal fluxgate, two-domain.

\section{INTRODUCTION}

$\mathbf{O}$ RTHOGONAL fluxgate was patented in 1951; this principle was almost forgotten as the mainstream large-size parallel fluxgate has shown lower noise and better stability. Orthogonal fluxgates based on microwires and planar microstructures reappeared recently; these devices have a good potential for miniaturization as they need no excitation coil-the sensor is excited by the current flowing through the core. This requires high currents to achieve full core saturation. The favorable design is a non-magnetic conductor covered by a magnetically soft film. The conductor is either a circular wire [1], [2] or rectangular rod [3]. In such case the magnetic core on the surface of the conductor is better saturated by the excitation current [4]. The orthogonal fluxgate is more sensitive than the "off-diagonal GMI" (or IWE) mode [5]. The working mechanism of the orthogonal fluxgate has not been fully understood yet.

Fluxgates work on second and sometimes higher-order even harmonic frequencies. Paperno has recently shown that in some cases the sensor noise in fundamental mode was lower than the noise of the same sensor in the 2nd harmonic mode [6]. He holds a noise record for transverse fluxgate, which is $20 \mathrm{pT} / \sqrt{ } \mathrm{Hz}$ at 1 $\mathrm{Hz}$. The poor offset temperature stability of this device was improved by periodical bias switching [7]. Temperature coefficient of the open-loop sensitivity can be compensated from 6500 to $100 \mathrm{ppm} / \mathrm{K}[8]$.

\section{PRIMdAhl's Model}

In [9] Primdahl proposed a simple model describing the rotation of the magnetization $\mathrm{M}$, due to the circumferential magnetic field $\mathrm{H}_{\phi}$. That model was based on the assumption of isotropy of the magnetic material; the first hypothesis Primdahl made, was the collinearity of B and H. Fundamental mode transverse fluxgate was later analysed by Sasada [10]; he has shown that this device cannot work without anisotropy. However, in this paper we concentrate on fluxgate which is saturated by excitation in both polarities, so that the sensor output is on the second harmonic.

Primdahl's model cannot be applied to the second-harmonic mode orthogonal fluxgates with electrodeposited magnetic wires. Measurement of the circumferential and longitudinal

Digital Object Identifier 10.1109/TMAG.2008.2003505

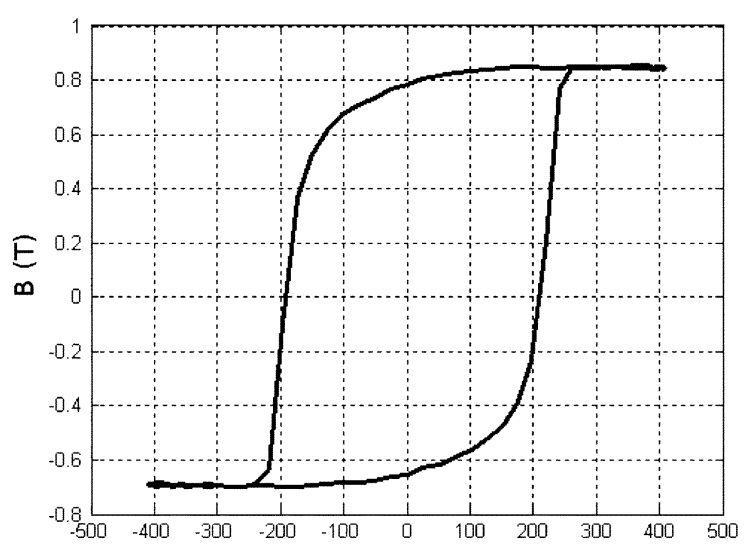

a) $\mathrm{H}(\mathrm{A} / \mathrm{m})$

b)

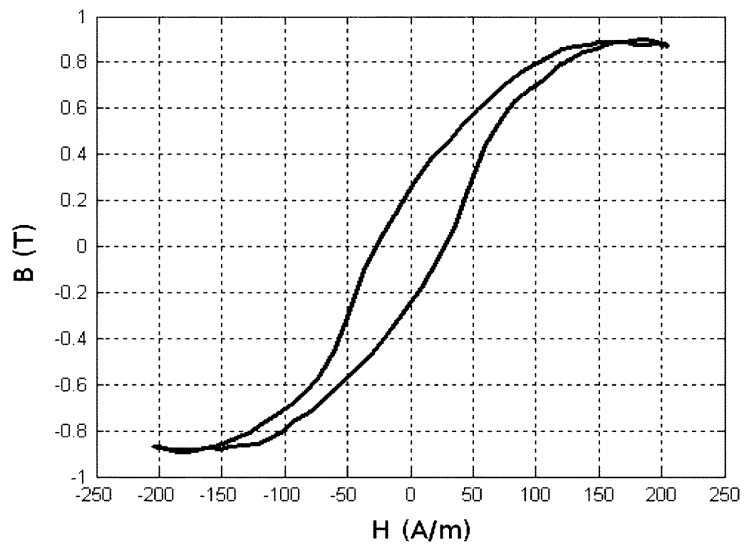

Fig. 1. $\mathrm{B}(\mathrm{T})-\mathrm{H}(\mathrm{A} / \mathrm{m})$ loops in longitudinal (a) and circumferential (b) direction.

B-H loops performed as explained in [11] on wire core electrodeposited by Atalay [12] revealed significant anisotropy [as seen from Fig. 1(a) and (b)]. We will further show that Primdahl's isotropic model cannot explain some typical features of the gating curves. Gating curve is a dependence of the longitudinal flux on circumferential excitation field $\mathrm{H}_{\phi}$.

Let us assume that the cylindrical core is subjected to longitudinal measured field $\mathrm{H}_{\mathrm{z}}$ and simultaneously to the circumferential excitation field $\mathrm{H}_{\phi}$. According to this model the magnetization process has two stages during increasing $\mathrm{H}_{\phi}$ : in the first stage $\mathrm{M}$ and $\mathrm{B}$ increase and simultaneously rotate from longitudinal towards circumferential direction. In the second phase B and $\mathrm{M}$ are saturated and only rotation of the magnetization occurs. Since the material is considered isotropic, in the first stage the magnetization $\mathrm{M}_{\mathrm{Z}}$ in longitudinal direction is constant, as it 


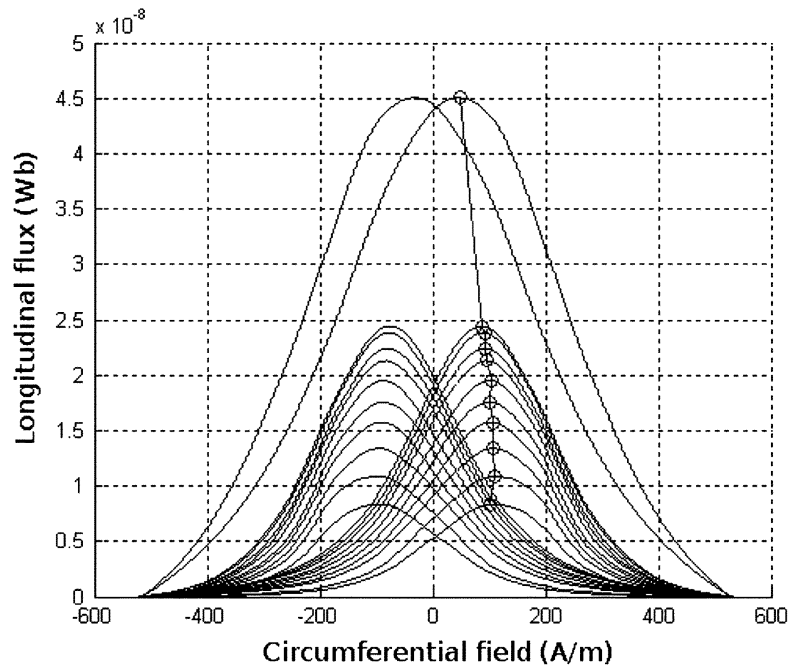

Fig. 2. Location of the maxima of the gating curve, for different value of measured longitudinal field $\mathrm{H}_{\mathrm{Z}}(2-20 \mathrm{~A} / \mathrm{m}$, step $2 \mathrm{~A} / \mathrm{m}$ and $300 \mathrm{~A} / \mathrm{m})$.

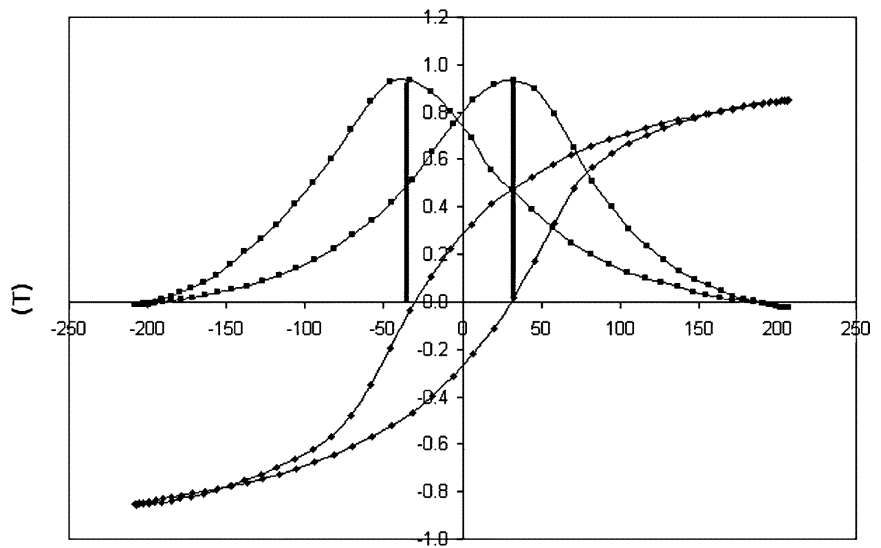

Circumferential field $(\mathrm{A} / \mathrm{m})$

Fig. 3. $B(T)-H(A / m)$ circumferential loop and gating curve (unscaled to allow comparison). We can observe that the maxima of the gating curve occur at coercivity in circumferential direction.

is proportional to the constant $\mathrm{H}_{\mathrm{Z}}$. Only in the second phase $\mathrm{M}_{\mathrm{Z}}$ decreases, inducing voltage in the pick-up coil.

In this model the fields are simply added: the higher is $\mathrm{H}_{\mathrm{Z}}$, the lower is the value of $\mathrm{H}_{\phi}$ necessary to saturate the core. From that we would expect the location of the maxima in the gating curve decreasing for increasing values of $\mathrm{H}_{\mathrm{Z}}$. We observed that that this is not true in the linear range of the fluxgate; Fig. 2 shows that the maxima of the gating curve occur at a constant value of $\mathrm{H}_{\phi}$, while changing $\mathrm{H}_{\mathrm{Z}}$ from $2 \mathrm{~A} / \mathrm{m}$ to $20 \mathrm{~A} / \mathrm{m}$.

The distance between the maxima changes only for high field values (i.e., comparable with the saturation field in circumferential direction). The observed distance between the peaks of the gating curve for small longitudinal field corresponds to the coercivity in circumferential direction (Fig. 3). Finally, we can assume this model can predict the tails of the gating curve, but it does not give a complete description of the mechanism underling the orthogonal fluxgates with thin-film core.

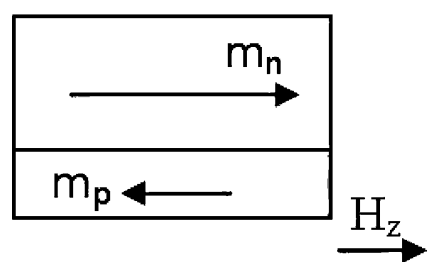

Fig. 4. Two-domain structure. $\mathrm{H}_{\mathrm{Z}}$ determines a domain wall movement.

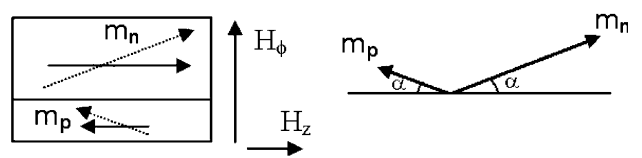

Fig. 5. Rotation of the magnetic moments of two-domains due to circumferential field $\mathrm{H}_{\phi}$.

\section{Two-DomaIn MODEL}

In our new model we assume the presence of easy axis of anisotropy in longitudinal direction, as typically found in electrodeposited wires. Our model consists of two longitudinal domains with opposite direction of magnetization. The longitudinal field $\mathrm{H}_{\mathrm{z}}$ causes domain wall movement from the central position: the domain with magnetization antiparallel to $\mathrm{H}_{\mathrm{z}}$ becomes smaller (Fig. 4).

When we apply circumferential field $\mathrm{H}_{\phi}$ (generated by the current flowing in the wire), the directions of the domains change in order to achieve the minimum of the total energy (1):

$$
\begin{aligned}
E_{m_{p, n}} & =E_{A}+E_{H} \\
& =\frac{1}{2} \mu_{0} m_{p, n} H_{C} \sin ^{2} \delta-m_{p, n} H_{\phi} \cos \varphi
\end{aligned}
$$

where $E_{A}$ is the anisotropy energy, $E_{H}$ is the energy related to the circumferential field $\mathrm{H}_{\phi}, \mathrm{H}_{\mathrm{C}}$ is the anisotropy field (in $\mathrm{Z}$ direction); $\delta$ is the angle between the magnetic moment and $\mathrm{H}_{\mathrm{C}}, \varphi$ is the angle between the magnetic moment and $\mathrm{H}_{\mathrm{C}}$. In (1) we did not include the energy term related to $\mathrm{H}_{Z}$, because in the linear range of the fluxgate $\mathrm{H}_{\mathrm{Z}} \ll \mathrm{H}_{\phi}$. Therefore we can neglect the effect of $\mathrm{H}_{Z}$ on the energy balance which determines the direction of the moment.

Since $\varphi=\pi / 2-\delta$ we can rewrite (1) as follows

$$
\begin{aligned}
E_{m_{p, n}} & =E_{A}+E_{H} \\
& =\frac{1}{2} \mu_{0} m_{p, n} H_{C} \sin ^{2} \delta-m_{p, n} H_{\phi} \sin \delta
\end{aligned}
$$

Then we calculate the derivative of (2) with the respect of $\delta$ and find its root $\alpha$ where the energy is minimum. Both the domains $m_{p}$ and $m_{n}$ will rotate by $\alpha$ Fig. 5) with opposite sign, because of the symmetrical properties of the trigonometric functions in (2).

In any case $\alpha$ is such that the magnetic moments will direct between the longitudinal direction $\mathrm{Z}$ (easy axis) and $\mathrm{H}_{\phi}$. This is due to the fact that the first term in (2) tries to pull the moment to circumferential direction (i.e., parallel to $\mathrm{H}_{\phi}$ ) whereas 


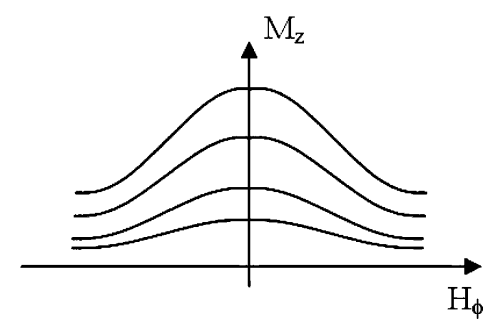

Fig. 6. Gating curve of orthogonal fluxgate expected from the two-domain model, in case of linearity in circumferential direction.

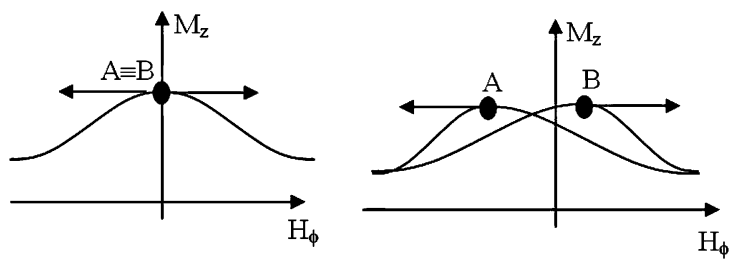

Fig. 7. Evolution of gating curve expected from the two-domain model, taking into account the hysteresis in circumferential direction.

the second term of (2) contributes to push the moment to longitudinal direction (i.e., parallel to $\mathrm{H}_{\mathrm{Z}}$ ). In the longitudinal direction we obtain net magnetization $\mathrm{M}_{\mathrm{z}}$ due to the difference between the magnetic moments projected into the $\mathrm{z}$ axis:

$$
\begin{aligned}
M_{Z} & \equiv m_{n} \cos \alpha-m_{p} \cos \alpha \\
& =\left(m_{n}-m_{p}\right) \cos . \alpha
\end{aligned}
$$

The term $\left(m_{n}-m_{p}\right)$ is the difference between the moments, due to $\mathrm{H}_{\mathrm{Z}}$, and it does not depend on $\mathrm{H}_{\phi}$. On the contrary $\alpha$ changes with $\mathrm{H}_{\phi}$.

We can see that $\mathrm{M}_{\mathrm{z}}$ is maximum when the moments have longitudinal direction $(\cos \alpha=1)$, and it decreases when $\mathrm{H}_{\phi}$ makes them rotate towards circumferential direction. The resulting gating curve is shown in Fig. 6 .

However we must take into the account also the hysteresis in circumferential direction. We can detect the instant when the two domains $m_{p}$ and $m_{n}$ lay horizontally from the circumferential B-H loop. This occurs when the circumferential flux $\mathrm{B}_{\phi}$ is zero, i.e., for $\mathrm{H}_{\phi}$ equal to the circumferential coercivity. If $\alpha=0$ we obtain a maximum in the gating curve as previously explained. This occurs twice per period, both for negative and positive coercivity. That is why the resulting gating curve has two peaks in correspondence of the coercivity in circumferential direction, as proven by the measurements (Fig. 3).

Basically the presence of hysteresis in circumferential B-H loop splits the maximum in the gating curve at $\mathrm{H}_{\phi}=0$ into two maxima which occur at positive and negative circumferential coercivity (Fig. 7).

\section{VerificATION OF THE PROPOSED MODEL}

The proposed model is based on simplified energy (2). In this equation we do not consider the whole magnetic field $\mathrm{H}$ resulting from the composition of $\mathrm{H}_{Z}$ and $\mathrm{H}_{\phi}$. On the contrary

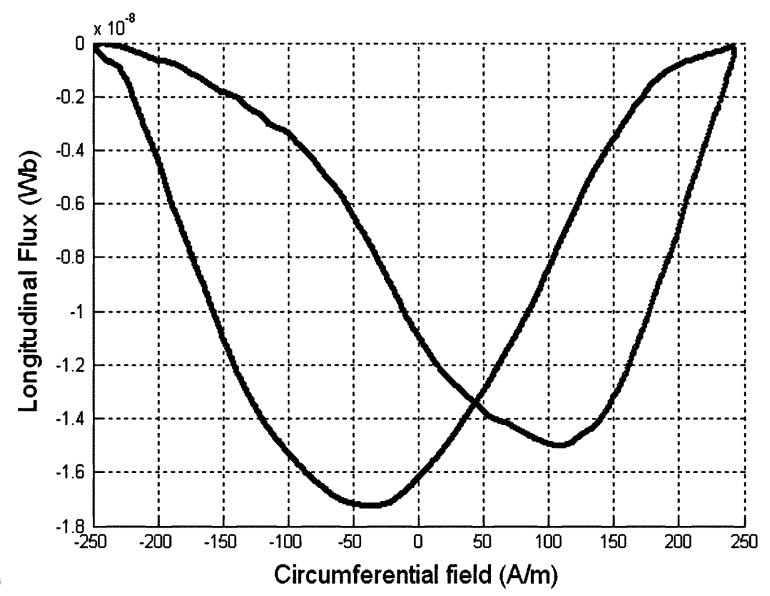

a)

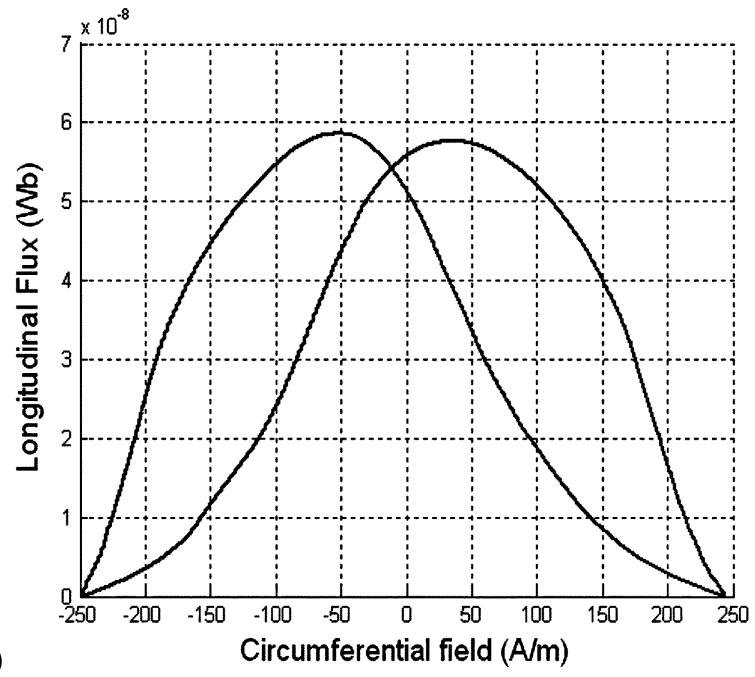

Fig. 8. Gating curve measured at $\mathrm{H}_{\mathrm{Z}}=4 \mathrm{~A} / \mathrm{m}$ before (a) and after (b) applying $\mathrm{H}_{\mathrm{Z}}=-300 \mathrm{~A} / \mathrm{m}$.

we keep apart the effect of $\mathrm{H}_{\mathrm{Z}}$ and $\mathrm{H}_{\phi}$. In fact we suppose that $\mathrm{H}_{\phi}$ makes only the domains rotate whereas $\mathrm{H}_{\mathrm{Z}}$ only moves the domain wall. This means that the height of the peaks at $\alpha=0$ is proportional to the longitudinal magnetization due to $\mathrm{H}_{\mathrm{Z}}$. Basically, in (3) $\mathrm{H}_{\phi}$ acts on the term $\cos \alpha$ whereas $\mathrm{H}_{\mathrm{Z}}$ acts on the term $\left(m_{n}-m_{p}\right)$.

In order to verify this hypothesis we conduct the following experiment.

We measured the gating curve for low $\mathrm{H}_{\mathrm{Z}}(4 \mathrm{~A} / \mathrm{m})$ while the $\mathrm{H}_{\phi \mathrm{pk}}$ was relatively low $(\sim 250 \mathrm{~A} / \mathrm{m})$. The gating curve was measured twice; between the first and the second measurement we applied $\mathrm{H}_{\mathrm{Z}}=-300 \mathrm{~A} / \mathrm{m}$ in longitudinal direction.

Fig. 8(a) and (b) shows the measured gating curves. We can observe that the height of the peaks drastically change. Only after we apply a magnetic field $\mathrm{H}_{\mathrm{Z}}=300 \mathrm{~A} / \mathrm{m}$ the gating curve returns to the same shape shown in Fig. 8(a).

This clearly shows that the height of the peaks is determined by the magnetization in longitudinal direction which is due to $\mathrm{H}_{Z}$. We can understand better this experiment if look to the longitudinal B-H loop (Fig. 9). The first gating curve (Fig. 8(a)) is measured at the point 1 of the B-H longitudinal loop, with $\mathrm{H}_{\mathrm{Z}}=4 \mathrm{~A} / \mathrm{m}$. Then $\mathrm{H}_{\mathrm{Z}}=-300 \mathrm{~A} / \mathrm{m}$ is applied (point 2), and 


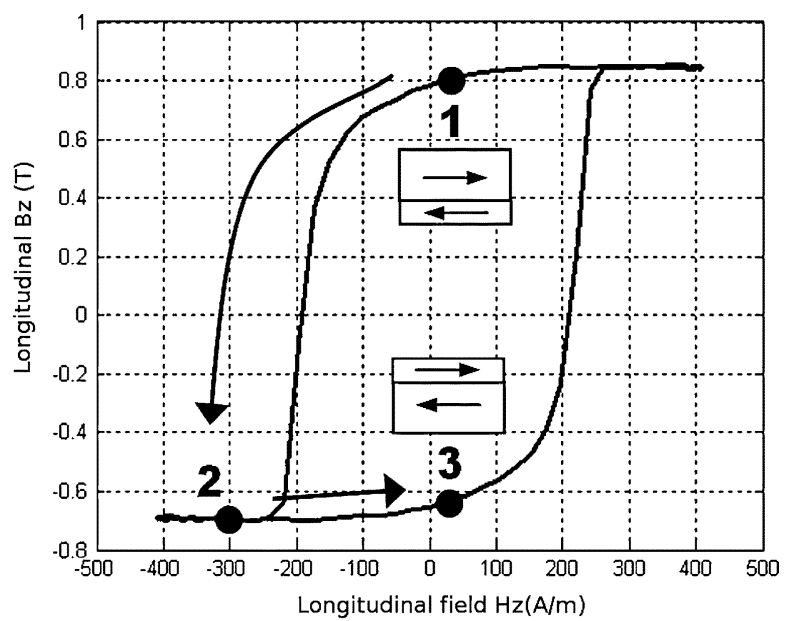

Fig. 9. Influence of magnetization process in longitudinal direction on the height of the B-H loops.

finally $\mathrm{H}_{\mathrm{Z}}$ gets back to $4 \mathrm{~A} / \mathrm{m}$ (point 3 ) which gives the second gating curve [Fig. 8(b)]. The process is clearly reversed when $\mathrm{H}_{\mathrm{Z}}=300 \mathrm{~A} / \mathrm{m}$ is applied.

If higher current flows in the wire, the generated $\mathrm{H}_{\phi}$ can reach a value high enough to overcome the remanence in longitudinal direction, due to $\mathrm{H}_{Z}=-300 \mathrm{~A} / \mathrm{m}$. In this case the wire is fully saturated in circumferential direction and the output response of the orthogonal fluxgate will be linear.

Finally we can state that the main role of $\mathrm{H}_{Z}$ is causing a domain wall movement; this gives a net magnetization in $\mathrm{Z}$ direction which is proportional to the height of the peaks in the gating curve.

Another proof of the proposed model is the correspondence of the peaks of the gating curve to the coercivity in circumferential direction (Fig. 3). As expected the locations of the maxima do not change for different values of $\mathrm{H}_{Z}$ (Fig. 2). Nevertheless we can notice this is not true anymore when $\mathrm{H}_{Z}$ reaches values out of the linear range. In fact for high $\mathrm{H}_{Z}$ the locations of the maxima in the gating curve get significantly closer (their modules decrease). This phenomenon typically occurs when $\mathrm{H}_{Z}$ is of the comparable size with $\mathrm{H}_{\phi}$.

This is clearly explained by the fact that under this circumstance we cannot neglect the contribution of $\mathrm{H}_{\mathrm{Z}}$ in the energy (1). Solution of (1) could be found even for such case, however this is out of our interests since this phenomenon is related to the non-linear range of the sensor. We can just notice that if we consider also $\mathrm{H}_{\mathrm{Z}}$ in (1) it is natural to expect a decrement of the minimum energy angle $\alpha$. The higher is $\mathrm{H}_{\mathrm{Z}}$ the more the domains are pushed to $\mathrm{Z}$ axis and consequently the B-H loop in circumferential direction results stretched. In this condition it is natural to expect a decrement of the module of the maxima in the gating curve.

\section{CONCLUSION}

In this paper we have proposed a new model of orthogonal fluxgate which is based on two-domain structure. We have given experimental proof of the fact that the model better suits to the orthogonal fluxgate made by microwires than the former model proposed by Primdahl [9].

This model can be also used to understand the coil-less fluxgate [13] mechanism. It is sufficient to consider helical anisotropy induced by torque and apply the same principle explained here; however such study is out of the scope of the present paper.

\section{ACKNOWLEDGMENT}

The work was supported by the research program No. MSM6840770015 "Research of Methods and Systems for Measurement of Physical Quantities and Measured Data Processing" of the CTU in Prague sponsored by the Ministry of Education, Youth and Sports of the Czech Republic.

The authors would like to thank S. Atalay and his team for the wires used for this research.

\section{REFERENCES}

[1] J. Fan, X. P. Li, and P. Ripka, "Low power orthogonal fluxgate sensor with electroplated Ni80Fe20/Cu wire," J. Appl. Phys., vol. 99, p. 08B311, 2006

[2] C. Petridis et al., "Ni-Fe thin film coated Cu Wires for field sensing applications," Sensor Lett., vol. 5, no. 1, pp. 93-97, Mar. 2007.

[3] O. Zorlu, P. Kejik, and R. S. Popovic, "An orthogonal fluxgate-type magnetic microsensor with electroplated permalloy core," Sens. Actuators $A$, vol. 135, pp. 43-49, 2007

[4] A. García, C. Morón, E. Tremps, F. J. Maganto, and J. L. Enríquez, "Orthogonal fluxgate of low noise by electrodeposited multilayers amorphous wires," Sensor Lett., vol. 5, pp. 212-214, 2007.

[5] P. Kollu, Ch. Kim, S. S. Yoon, and Ch. Kim, "Highly sensitive giant magneto impedance sensor with LC pick-up circuit," Sensor Lett., vol. 5, pp. 157-161, 2007.

[6] E. Paperno, "Suppression of magnetic noise in the fundamental-mode orthogonal fluxgate sens," Actuator A-Phys., vol. 116, pp. 405-409, 2004.

[7] K. Goleman and I. Sasada, "High sensitive orthogonal fluxgate magnetometer using a metglas ribbon," IEEE Trans. Magn., vol. 42, pp. 3276-3278, 2006.

[8] P. Anton, E. Paperno, A. Samohin, and I. Sasada, "Compensation of the thermal drift in the sensitivity of fundamental-mode orthogonal fluxgates," J. Appl. Phys., vol. 99, p. 08B305, 2006.

[9] F. Primdahl, "The fluxgate mechanism, part I: The gating curves of parallel and orthogonal fluxgates," IEEE Trans. Magn., vol. MAG-6, pp. 376-383, 1970

[10] I. Sasada, "Symmetric response obtained with an orthogonal fluxgate operating in fundamental mode," IEEE Trans. Magn., vol. 38, pp. 3377-3379, 2002.

[11] P. Ripka, M. Butta, M. Malatek, S. Atalay, and F. E. Atalay, "Characterization of magnetic wires for fluxgate cores," Sens. Actuators A: Phys., 2007, in print, doi:10.1016/j.sna.2007.10.008.

[12] F. E. Atalay, H. Kaya, and S. Atalay, "Magnetoimpedance effect in electroplated $\mathrm{NiFeRu/Cu}$ wire," J. Phys. D: Appl. Phys., vol. 39, pp. 431-436, 2006.

[13] M. Butta, P. Ripka, S. Atalay, F. E. Atalay, and X. P. Li, "Fluxgate effect in twisted magnetic wire," J. Magn. Magn. Mater., accepted for publication.

Manuscript received March 03, 2008. Current version published December 17, 2008. Corresponding author: M. Butta (e-mail: buttam1@ fel.cvut.cz). 